\title{
Left ventricular ejection fraction assessed by echocardiography and magnetic resonance imaging in acute anterior and non-anterior STEMI
}

\author{
Gert Klug ${ }^{*}$, Sebastian J Reinstadler, Hans-Josef Feistritzer, Bastian Pernter, Wolfgang-Michael Franz, Silvana Müller, \\ Bernhard Metzler
}

From 18th Annual SCMR Scientific Sessions

Nice, France. 4-7 February 2015

\section{Background}

We have previously shown a moderate agreement of echocardiography and cardiac magnetic resonance (CMR) in the assessment of left ventricular ejection fraction (LVEF) after acute ST-segment elevation infarction (STEMI). As ejection fraction after STEMI is of paramount prognostic importance we investigated the impact of infarct location on the agreement of echocardiography and CMR.

\section{Methods}

One-hundred and seventy-nine patients (mean age: $57 \pm$ 11 years, $\mathrm{n}=27$ female) with first acute STEMI were enrolled in this single-center registry study. Patients underwent CMR (median: 2.4 days) and echocardiography (median 3 days) within the first week after admission. LVEF was determined from short-axis slices with CMR and with a modified Simpson rule from apical 4chamber echo views. Infarct size was determined from late-gadolinium enhanced (LGE) CMR.

\section{Results}

Mean LVEF determined by echocardiography was $50 \pm$ $10 \%$ and $52 \pm 11 \%$ as determined by CMR (paired Wilcoxon test: $\mathrm{p}=0.021$ ). The correlation between echocardiography and CMR was moderate ( $\mathrm{r}: 0.492, \mathrm{p}<0.001)$. The correlation of LVEF with infarct size was $\mathrm{r}:-0.306$ (echocardiography) and r:-0.430 (CMR) respectively (both $\mathrm{p}<0.001)$. The agreement of echocardiography and CMR was higher in anterior STEMI $(n=73)(r: 0.629, p<0.001)$ compared to non-anterior STEMI $(\mathrm{n}=105)(\mathrm{r}: 0.279$, $\mathrm{p}=0.004)(\mathrm{z}$-score: $2.92, \mathrm{p}=0.003)$. The correlation of both methods with infract size was higher in anterior STEMI (CMR r:-0.588 and echocardiography r:-0.485, both $\mathrm{p}<0.001)$ than in non-anterior STEMI (CMR r:-0.293, $\mathrm{p}<0.001$ and echo $\mathrm{r}:-0.075, \mathrm{p}=\mathrm{NS}$ ) ( $\mathrm{z}$-scores -2.4 and -3.0 , both $\mathrm{p}<0.02)$.

\section{Conclusions}

LVEF by CMR is higher than estimated by echocardiography. The agreement of both methods is significantly higher in anterior STEMI than in non-anterior STEMI. Interestingly both methods show only weak to nonsignificant correlations of LVEF and infarct size in nonanterior STEMI. These results might help to select patients for CMR prior to therapeutic decisions based on LVEF after acute STEMI.

\section{Funding}

Austrian Society of Cardiology, MUI-Start.

Published: 3 February 2015

doi:10.1186/1532-429X-17-S1-P159

Cite this article as: Klug et al: Left ventricular ejection fraction assessed

by echocardiography and magnetic resonance imaging in acute

anterior and non-anterior STEMI. Journal of Cardiovascular Magnetic Resonance 2015 17(Suppl 1):P159. 